\title{
Childhood Cancer Survivorship and Late Effects - The Landscape in India in 2020
}

\author{
Ramandeep Arora ${ }^{1}$, Puneet Arora ${ }^{2}$, Rachna Seth $^{3}$, Sonal Sharma ${ }^{4}$, Chandan Kumar ${ }^{4}$, \\ Vandana Dhamankar ${ }^{5}$, PURNA KURKURE ${ }^{6}$, and Maya Prasad ${ }^{7}$ \\ ${ }^{1}$ Max Super-Speciality Hospital \\ ${ }^{2}$ Milann Fertility Centre \\ ${ }^{3}$ All India Institute of Medical Sciences \\ ${ }^{4}$ Cankids \\ ${ }^{5}$ Indian Cancer Society \\ ${ }^{6}$ Narayana Health SRCC Children's Hospital \\ ${ }^{7}$ Tata Memorial Hospital
}

May 15, 2020

\begin{abstract}
Survivorship care is a major area of focus in the holistic management of childhood cancer with current knowledge and information almost exclusively from high-income countries. In this review, we summarize the state of scientific knowledge, service delivery, advocacy initiatives and research efforts in this field in India. 21 single centre studies published till date (20 in the last decade) confirm some of the well-documented issues in childhood cancer survivors and also highlight the high prevalence of hepatitis $\mathrm{B}$ and hepatitis $\mathrm{C}$ infection in our survivors. Heterogeneity in methodology, outcome metrics and quality precludes drawing further conclusions and the ongoing multicenter Indian Pediatric Oncology Group study would address this. Besides the usual model of follow-up clinics in hospital settings, innovative models of service delivery led by not-for-profit organisations are being developed. Advocacy initiatives driven by survivors and support groups are also underway. All of these portend a promising future.
\end{abstract}

\begin{abstract}
Survivorship care is a major area of focus in the holistic management of childhood cancer with current knowledge and information almost exclusively from high-income countries. In this review, we summarize the state of scientific knowledge, service delivery, advocacy initiatives and research efforts in this field in India. 21 single centre studies published till date (20 in the last decade) confirm some of the well-documented issues in childhood cancer survivors and also highlight the high prevalence of hepatitis B and hepatitis C infection in our survivors. Heterogeneity in methodology, outcome metrics and quality precludes drawing further conclusions and the ongoing multicenter Indian Pediatric Oncology Group study would address this. Besides the usual model of follow-up clinics in hospital settings, innovative models of service delivery led by not-for-profit organisations are being developed. Advocacy initiatives driven by survivors and support groups are also underway. All of these portend a promising future.

Outcomes of childhood cancer have improved steadily and consistently over the last several decades and now more than eight out of 10 children with cancer in high-income countries are long-term survivors and considered cured of their disease. With an estimated half a million long-term survivors of childhood cancer in $\mathrm{USA}^{1}$ and a similar number in Europe ${ }^{2}$, survivorship practice and research are major areas of focus for
\end{abstract}


healthcare professionals, policy makers and patient advocates. Approximately, two of every three childhood cancer survivors will experience at least one late effect, and $40 \%$ may develop a "severe, disabling, or life-threatening condition" 30 years after cancer diagnosis. ${ }^{3}$ Not surprisingly, the focus of the pediatric oncology professionals has shifted for "Cure at all cost" to "Equivalent cure with minimal cost". Contemporary treatment protocols give considerable importance to minimizing long-term impacts of treatment while maintaining high cure rates.

In contrast to the progress seen in high income countries, low and middle income countries like India have continued to confront the triple challenges of high treatment abandonment, toxicity-related mortality and disease progression. ${ }^{4,5}$ Consequently improvements in survival are more modest and till recently, survivorship has not been a priority at service, research or policy level. ${ }^{6,7}$ The last few years have witnessed an increased momentum in issues related to survivors and survivorship. In this review we aim to summarize the current state of scientific knowledge, service delivery, advocacy initiatives and research efforts in this field in India.

\section{Review of published literature from India}

We identified references for this review through searches of PubMed using the following search terms or combinations of search terms: "Survivors", "Neoplasms", "India", "Child", "Late effects" and "Long-Term Follow Up". Reference lists of the shortlisted studies were reviewed to identify potentially relevant previous studies. Similarly Google Scholar was used to see citations of the shortlisted studies in order to identify potentially relevant subsequent studies. Each of the co-authors was also asked to review their own files to ensure that the literature search had been comprehensive. The final list was generated on the basis of originality and relevance to the scope of this review.

Summary of results of 21 studies $^{7-27}$ are tabulated (Table 1) and categorized by organ systems and late effects as used in the Children's Oncology Group Survivorship Guidelines. ${ }^{28}$ All except one ${ }^{7}$ of these studies, has been published in the last decade, highlighting the recent expansion in work in this area in India. These were all single centre studies with 15 of the 21 studies from All India Institute of Medical Sciences, New Delhi and Tata Memorial Hospital, Mumbai. Sample sizes ranged from 20 to 1285 with median of 119 patients. Median follow-up was 4.5 years (range $>1$ year to 12 years). Most studies lacked controls, ${ }^{7-14,17-21,23-27}$ which is important to quantify the increased risk of the problem as compared to the general population. Importantly, with few exceptions ${ }^{9,11,24}$, most studies had not examined dose-effect relationship of exposure or the effect of combined modality of treatment. Heterogeneity in methodology (of patient selection, defining outcomes and tools used) made it unfeasible to pool data and give a summative risk for each category. The data is hence displayed as a table to allow the reader to get an overview of the methodology and results.

Despite the above limitations, several important observations can be made. These studies confirmed some of the well-documented issues in childhood cancer survivors including the previously reported detrimental effects of cranial radiation on neurocognition ${ }^{9-12}$ and neuroendocrine axis ${ }^{11}$, of neck radiation on thyroid function ${ }^{7,10}$, and of alkylating agents on fertility. ${ }^{10,12,24,25}$ Contrary to published literature, ${ }^{28}$ a single study from India showed bone mineral density in leukemia survivors was similar to that of controls. ${ }^{22}$ The prevalence of overweight/obesity varied from $1 \%$ to $32 \%$ and may reflect the heterogeneity in patient selection, methodology and study design. ${ }^{10,12,19-23}$ For late-effects of the heart the duration of follow-up was not sufficient to conclude on the magnitude of increased risk. The risk of secondary malignancy was $0.7 \%$ to $1.8 \%$ with median follow-up of 4.7 to 12 years.

Studies from three major centres in India, which included patients treated in the $21^{\text {st }}$ century, also highlighted the specific challenge of transmitted viral infections with high prevalence of Hepatitis B (20\% to $54 \%$ ) and Hepatitis C (6\% to 25\%) infection in survivors. ${ }^{7,10,12}$ Blood product transfusion played a major role in this although they were likely to be other factors. ${ }^{12,29}$ Improvements in standards of screening of blood products has led to major decreases in transfusion transmitted viral-infections globally ${ }^{30}$ although the risk of acquiring these infections is still unacceptably high in low and middle income countries. ${ }^{31}$ Although, standards of testing including NAT testing have gradually improved, the progress has been patchy and 
$\operatorname{tardy}^{32}$

\section{Service delivery and models}

The burgeoning number of childhood cancer survivors in high income countries with their morbidity burden necessitated steady and sustained efforts in developing services for their needs. While there is variation in provision of these services, there is universal agreement on their need. ${ }^{33,34}$ Few specific services are worth highlighting.

Firstly, is the model of long-term follow-up (FU) care. There is no universally agreed "best" model and the choice depends on patient volumes, complexity of needs, resources available and travel distances. Some of these models include FU in primary pediatric oncology setting, transitioning to an adult oncology setting, specialized long-term FU clinic or shared care. ${ }^{34}$ The second noteworthy development is that of guidelines to support lifelong risk-based follow up for this population. A clear understanding of the association between therapeutic exposures and specific long-term complications, and an understanding of the magnitude of the burden of morbidity borne by childhood cancer survivors, has led to this. ${ }^{28,35}$ It is worth pointing out that the data supporting these guidelines have come exclusively from research done in high income countries and hence their relevance when applying to low-middle income countries remains to be established. The increasing use of Survivor Passports is another highlight in the service delivery of these survivors. ${ }^{36,37}$ These passports not only provide a summary of each survivor's clinical history, with detailed information about the original cancer and of treatments received, but also give personalised follow-up and screening recommendations based on guidelines thus ensuring high quality uniform care.

Surveys from high-income countries demonstrate service delivery for these survivors. ${ }^{38-41}$ Such information is absent in the Indian context. We recently conducted an online survey with the objective of determining the current service provided to childhood cancer survivors in India. ${ }^{42}$ It was sent to primary/senior consultants treating children with cancer in 86 centres in India.

Responses from $52(61 \%)$ centres in 24 cities have been received (private $46 \%$, public $31 \%$, charitable trust $23 \%)$. Upper age cut-off for FU of survivors was no age cut-off $(63 \%), 18$ years $(21 \%), 21$ years $(10 \%)$. Similarly, cut-off based on duration from end of treatment was no cut-off $(60 \%), 5$ years (38\%). In $90 \%$ of the centres, FU of survivors (more than 2 years off treatment) was in the regular pediatric oncology clinic while $10 \%$ had a dedicated clinic. The current level of service was thought to be inadequate or very inadequate $19 \%$, neither adequate nor inadequate by $56 \%$ of centres, and adequate or very adequate $25 \%$. The most important barriers were distance to clinic and patients/parents lack of knowledge about the need for FU.

Below are some examples of current models of service delivery to childhood cancer survivors in India

Model 1 - Follow up driven by healthcare establishments in hospital setting

As described above, these follow up clinics are multidisciplinary, mainly pediatric oncology physician led. They mainly cater to children and adolescents and follow-up duration is short. Some centres give survivorship summaries and 10-15 centres have dedicated survivorship clinics, most of whom have started in the last 5 years. Below are a couple of examples of wellestablished dedicated survivor clinics.

\section{Tata Memorial Hospital, Mumbai}

The After Completion of Therapy Clinic (ACT) Clinic at Tata Memorial Hospital, Mumbai was established in 1991, the first such clinic in India. From its inception, the functioning has been multidisciplinary with pediatric oncologist, psychologist and social worker. Additionally, over the years, there are dedicated weekly clinics for cardio-oncology and endocrinology follow-up of survivors of childhood cancer. Currently there are 
3000 survivors registered, of whom $60 \%$ follow-up regularly. The median age of these survivors is 18 years, with a median follow-up duration of 10 years. Owing to the longstanding nature of this clinic, survivors are predominantly adolescent and young adults, with over $2 / 3^{\text {rd }}$ of survivors being adults. Thanks to generous financial support from donors and liaison with Indian cancer society, the ACT clinic is now able to provide holistic support to its survivors.

\section{All India Institute of Medical Sciences, New Delhi}

The Pediatric Cancer Survivor Clinic of the department of Pediatrics, AIIMS is a weekly after treatment completion clinic where all children who have completed their treatment for the primary cancer are followed. The team at the clinic comprises of pediatric oncologists, nurses, counselors, dietician and non-governmental organization staff. On an average 150 new patients are registered into the clinic annually. A detailed counseling session is done with the family wherein the importance of long-term follow-up is explained. A bilingual After Treatment Completion Card is provided to all patients which records the treatment given (dates, types of treatment, cumulative doses) and possible late effects (Figure 1). ${ }^{43}$ Information regarding healthy lifestyle and vaccination is given. Girls are advised to take Human Papilloma Virus vaccine. Details of growth and disease status are captured and necessary tests conducted. A detailed psychosocial evaluation and intelligence quotient assessment are done for children who received central nervous system directed therapy in the form of chemotherapy/radiation. Information on social adjustment into the home, school and society are recorded. Information regarding education and peer relationships is acquired from all children and grades of disability ascertained. Regular follow-up is scheduled.

Model 2 - Follow-Up Driven by Not for Profit Organisations in hospital or community settings

Identifying the deficiency in survivorship services, a couple of not for profit organisations have stepped in. Besides managing medical issues, they are also able to provide social support, engage survivors through peer groups and advocate.

\section{Cankids Survivor Passport2Life Clinics}

Cankids, a national society for Change for Childhood Cancer, launched the Passport2Life (P2L) Project in recognition of the lack of information available to childhood cancer survivors and the absence of systematic follow-up. Through the P2L project, Cankids aims to help the enrolled survivors to know the potential risk of late side effects and how they can cope up with them.

Cankids has developed two different strategies for conducting the survivor P2L clinics to ensure that the maximum numbers of survivors are covered, and at the same time accommodating the needs of treating doctors at different hospitals. Strategy-1 (P2L - C2H) currently running in 12 locations: - Whereas "C" denotes Cankids and " $\mathrm{H}$ " denotes treating Hospital. Cankids collaborates with the treating hospitals to run the Passport2Life clinic and workshops in the respective hospital. Strategy-2 (P2L- C2S) currently running in 2 locations: - Whereas "C" denotes Cankids and "S" denotes Survivor. Cankids conducts the P2L clinics and workshops for those survivors whose hospital are not providing late effect clinic, or survivors not able to reach out the treating centres, or survivors are older and don't want to go back to the pediatric unit or survivors have moved to other cities.

A document (Survivor Passport) is completed and provided to the survivor (Figure 1). Details are also entered onto www.survivorp2l.org, which functions as an online passport and community portal.

\section{Project PICASSO of Indian Cancer Society}

As early as 1951, Dr. DJ Jussawalla and Mr. Naval Tata started the first voluntary, not for profit, national organization, the Indian Cancer Society (ICS). It was initiated to spread awareness about cancer, initiate early detection, promote cure and survivorship of those affected with this disease.

Survivorship and rehabilitation vertical of ICS has developed and initiated the survivorship module PICASSO (Partnership In CAncer SurvivorShip Optimization) in 2017. Taking inspiration from the ACT clinic at Tata 
Memorial Hospital and involving Ugam (support group of survivors), PICASSO is holistic rehabilitation module being implemented in partnership with pediatric cancer units. The objective is to facilitate the pediatric cancer units to start hospital based ACT Clinic where pediatric oncologist does medical assessments and psychosocial care is taken care of by ICS for holistic (medical and psychosocial) care of survivors. The purpose is to decrease the loss to long term FU of survivors, to improve their quality of life by innovating and developing effective rehabilitation programs, and to facilitate diverse networks of support groups that could facilitate the integration of survivors in the mainstream of society. Through five partners, 697 survivors have been enrolled in PICASSO from November 2017 till Aug 2019.

\section{Advocacy Initiatives}

Childhood cancer survivors are the best advocates for their cause. As a survivor rightly said "Nobody cares about me more than me ". ${ }^{44}$ Their voice can amplify gaps in their care and galvanize efforts in service, policy and research. Nearly two decades ago, the development of the National Action Plan for Childhood Cancer in USA brought together several stakeholders including civil society, parents and survivors. ${ }^{45}$ They specifically made recommendations for long-term care and survivorship including identifying concerns, developing educational materials, receiving care, setting standards and developing a database. International and National childhood cancer survivor groups have continued to advocate for change in policy and service. ${ }^{46,47}$ Similar recent efforts have been made by survivors of childhood cancer in India, especially Kidscan Konnect (KCK) from Cankids and Ugam, which is part of ICS.

\section{Cankids KCK}

Cankids set up the childhood cancer teenage and young adult survivor group called KCK in 2006 (https://www.facebook.com/kidscankonnect.in/). Today KCK has a membership of over 900 survivors, with 10 chapters in cities across India. Childhood cancer survivors join as members (age has to be 13 years and above) and are groomed to be chapter heads, leaders, and then mentors.

Cankids KCK advocacy initiatives are at hospital, state, national and international level. At hospital level, it advocates for survivorship groups, regular survivor forums, and dedicated survivorship clinics (Figure 2). Survivor coordinators cum data managers are provided for the P2L Clinics. At state and national level, the KCK group actively engages in creating awareness and organizes and participates in access to care camps and programs, scripts and enacts its own street plays for patient care and survivorship issues. Survivor Week is celebrated in June each year. Four national survivor conferences have been organized in 2010, 2011, 2015 and 2018. In 2016-17 national debates on the Right to Education were organized as survivors campaigned for inclusion in the Persons with Disability Act, and a petition was submitted in September 2017 to the Government of India. On the international level, KCK members are on the Asia Childhood Cancer Survivor Network Management team and are active members and participants in the Childhood Cancer International Survivor Network and regularly present on developments on survivorship in India. In 2019, Ritu Bhalla Girl Child Cancer Ambassador of India, gave the Opening address at the World Health Organization South East Asia Regional Office Workshop to accelerate cancer prevention and control in South East Asia held in New Delhi on 25th -26th June 2019 (Figure 2).

ICS and Ugam

On the $7^{\text {th }}$ of June 2009 on the occasion of Cancer Survivors Day, a small group of motivated childhood cancer survivors came together with a focused vision of self-empowerment. Ugam was then born and formally launched under the survivorship banner of ICS. Ugam means "To Rise", underscoring the determination of childhood cancer survivors, to rise above all obstacles in life and be victors https://www.indiancancersociety.org/events/ugam.aspx.

Subsequently their activities over a decade have included participation in International Relay For Life (a 
franchised cancer awareness event of American Cancer Society) Mumbai Marathon (Figure 2), awareness activities, sharing stories and giving hope to the parents and children with cancer who are undergoing treatment and residing at home away from home such as St Jude Child Care centres. Ugam members have initiated advocacy for providing health insurance to survivors of childhood cancer and will help survivors to get the insurance cover rightfully. Another area of concern is matrimonial prospects for survivors who are now adults and willing to settle down in family life but face societal resistance. Ugam is a torchbearer and advocate for these burning issues at societal, non-governmental and governmental level.

Ugam is also an active member of the Asian network of survivors under the umbrella of Childhood Cancer International (CCI) as. Ugam members have been regularly awarded fellowships since 2013 to participate in Annual conference of CCI alongside the Annual conference of International Society of Pediatric Oncology.

\section{Research}

Much of our knowledge has come from research on large cohorts of childhood cancer survivors who have been followed-up meticulously and painstakingly to gain insight into their health problems and psychosocial issues. ${ }^{48}$ Till recently similar cohorts have not existed in low-middle income countries. The growth of the Indian Pediatric Oncology Group (InPOG) has provided the platform for multicenter co-operative group research in India. ${ }^{49}$ One of the earliest studies in the InPOG portfolio has been InPOG-LE-16-01 study. The aim of the study is to establish a multi-centre (ultimate goal - nation-wide) registry of children completing treatment for childhood cancer and in complete remission for a period of at least 2 years. As of April 2020, this study is open in 14 centres and has recruited 1801 patients. Future plans include growing this cohort by inviting more centres to be part of this study and continue enrollment in existing centres. In parallel, several research proposals are in development, which would aim to answer the first generation of research questions using childhood cancer survivors of Indian in this cohort.

With the changing role of patients in health research, KCK survivors are also leading research. Such involvement ensures that relevant questions are answered, and study results are disseminated and utilized. Currently four survivor led research projects are underway including Passport2Life, fertility status, nutrition status and stigma, bias and empowerment study in the girl childhood cancer survivor

\section{Conclusion}

After a slow start, there is now increasing momentum in work done being for childhood cancer survivors in India. Although there remains a long way to go, this progress is happening on multiple fronts, has engagement of various stakeholders, and there are early signs of collaboration. All of these portend a promising future.

Conflict of Interest: All authors declare that they have no conflict of interest

Consent Statement: All the individuals in Figure 2 have given permission to use their image in the manuscript.

\section{References}

1. Robison LL, Hudson MM. Survivors of childhood and adolescent cancer: life-long risks and responsibilities. Nat Rev Cancer 2014;14:61-70.

2. Hjorth L, Haupt R, Skinner R, Grabow D, Byrne J, Karner S, Levitt G, Michel G, van der Pal H, Bárdi E, Beck JD, de Vathaire F, Essig S, Frey E, Garwicz S, Hawkins M, Jakab Z, Jankovic M, Kazanowska B, Kepak T, Kremer L, Lackner H, Sugden E, Terenziani M, Zaletel LZ, Kaatsch P; PanCare Network. Survivorship after childhood cancer: PanCare: a European Network to promote optimal long-term care. Eur J Cancer 2015;51:1203-1211.

3. Oeffinger KC, Mertens AC, Sklar CA, Kawashima T, Hudson MM, Meadows AT, Friedman DL, Marina N, Hobbie W, Kadan-Lottick NS, Schwartz CL, Leisenring W, Robison LL; Childhood Cancer Survivor 
Study. Chronic health conditions in adult survivors of childhood cancer. N Engl J Med 2006;355:15721582.

4. Arora RS, Eden TO, Kapoor G. Epidemiology of childhood cancer in India. Indian J Cancer 2009;46:264-273.

5. Arora RS, Eden T, Pizer B. The problem of treatment abandonment in children from developing countries with cancer. Pediatr Blood Cancer 2007;49:941-946.

6. Kurkure PA, Achrekar S, Uparkar U, Dalvi N, Goswami S. Surviving childhood cancer: what next? Issues under consideration at the After Completion of Therapy (ACT) clinic in India. Med Pediatr Oncol 2003;41:588-589.

7. Kurkure P, Achrekar S, Dalvi N, Goswami S. Childhood cancer survivors-living beyond cure. Indian J Pediatr 2003;70:825-828.

8. Gupta T, Jalali R, Goswami S, Nair V, Moiyadi A, Epari S, Sarin R. Early clinical outcomes demonstrate preserved cognitive function in children with average-risk medulloblastoma when treated with hyperfractionated radiation therapy. Int J Radiat Oncol Biol Phys 2012;83:1534-1540.

9. Jalali R, Mallick I, Dutta D, Goswami S, Gupta T, Munshi A, Deshpande D, Sarin R. Factors influencing neurocognitive outcomes in young patients with benign and low-grade brain tumors treated with stereotactic conformal radiotherapy. Int J Radiat Oncol Biol Phys 2010;77:974-979.

10. Rajendranath R, Veeraiah S, Ramesh A, Sagar TG. Late effects of treatment in survivors of childhood cancer from a tertiary cancer center in South India. South Asian J Cancer 2014;3:60-65.

11. Jalali R, Gupta T, Goda JS, Goswami S, Shah N, Dutta D, Krishna U, Deodhar J, Menon P, Kannan S, Sarin R. Efficacy of Stereotactic Conformal Radiotherapy vs Conventional Radiotherapy on Benign and Low-Grade Brain Tumors: A Randomized Clinical Trial. JAMA Oncol 2017;3:1368-1376.

12. Seth R, Singh A, Guru V, Chawla B, Pathy S, Sapra S. Long-term follow-up of retinoblastoma survivors: Experience from India. South Asian J Cancer 2017;6:176-179.

13. Seth R, Singh A, Seth S, Sapra S. Late effects of treatment in survivors of childhood cancers: A single-centre experience. Indian J Med Res 2017;146:216-223.

14. Jain P, Gulati S, Seth R, Bakhshi S, Toteja GS, Pandey RM. Vincristine-induced neuropathy in childhood ALL (acute lymphoblastic leukemia) survivors: prevalence and electrophysiological characteristics. J Child Neurol 2014;29:932-937.

15. Batra A, Kumari M, Paul R, Patekar M, Dhawan D, Bakhshi S. Quality of Life Assessment in Retinoblastoma: A Cross-Sectional Study of 122 Survivors from India. Pediatr Blood Cancer 2016;63:313-317.

16. Batra A, Kain R, Kumari M, Paul R, Dhawan D, Bakhshi S. Parents' Perspective of Quality of Life of Retinoblastoma Survivors. Pediatr Blood Cancer 2016;63:1287-1289.

17. Batra A, Pushker N, Venkatesh P, Arora T, Tewari R, Bakhshi S. Long-term visual outcomes in intraocular retinoblastoma with eye preservation. Clin Transl Oncol 2016;18:1034-1038.

18. Batra A, Thakar A, Bakhshi S. Ototoxicity in retinoblastoma survivors treated with carboplatin based chemotherapy: A cross-sectional study of 116 patients. Pediatr Blood Cancer 2015;62:2060.

19. Prasad M, Arora B, Chinnaswamy G, Vora T, Narula G, Banavali S, Kurkure P. Nutritional status in survivors of childhood cancer: Experience from Tata Memorial Hospital, Mumbai. Indian J Cancer. 2015;52:219-223.

20. Srivastava R, Batra A, Tyagi A, Dhawan D, Ramakrishnan L, Bakhshi S. Adiponectin correlates with obesity: A study of 159 childhood acute leukemia survivors from India. Indian J Cancer 2015;52:195197.

21. Mohapatra S, Bansal D, Bhalla AK, Verma Attri S, Sachdeva N, Trehan A, Marwaha RK. Is there an increased risk of metabolic syndrome among childhood acute lymphoblastic leukemia survivors? A developing country experience. Pediatr Hematol Oncol 2016;33:136-149.

22. Jain S, Jain S, Kapoor G, Virmani A, Bajpai R. No impact of disease and its treatment on bone mineral density in survivors of childhood acute lymphoblastic leukemia. Pediatr Blood Cancer 2017;64:e26271.

23. Mahalingam S, Bhat KG, Dhulipalli A, Ramaswamy S. Obesity, Dyslipidemia and Insulin Resistance in Survivors of Childhood Cancer. Iranian Journal of Pediatric Hematology \& Oncology 2019;9:1-8.

24. Arora PR, Misra R, Mehrotra S, Mittal C, Sharma S, Bagai P, Arora RS. Pilot initiative in India to 
explore the gonadal function and fertility outcomes of a cohort of childhood cancer survivors. J Hum Reprod Sci 2016;9:90-93.

25. Kurkure P, Prasad M, Dhamankar V, Bakshi G. Very small embryonic-like stem cells (VSELs) detected in azoospermic testicular biopsies of adult survivors of childhood cancer. Reprod Biol Endocrinol 2015;13:122.

26. Batra A, Patekar M, Bakhshi S. Short stature in retinoblastoma survivors: a cross-sectional study of 138 patients. Clin Transl Oncol. 2016;18:381-384.

27. Vyas C, Jain S, Kapoor G. Therapy Related AML/MDS Following Treatment for Childhood Cancer: Experience from a Tertiary Care Centre in North India. Indian J Hematol Blood Transfus 2018;34:7882.

28. Children's Oncology Group. Long-Term Follow-Up Guidelines for Survivors of Childhood, Adolescent and Young Adult Cancers, Version 4.0. Monrovia, CA: Children's Oncology Group; October 2013; Available on-line: www.survivorshipguidelines.org.

29. Arora B, Joshi YK, Salhan RN, Arya LS, Prakash S. Transfusion-associated hepatitis in children with hematologic malignancies in Northern India. Med Pediatr Oncol 2003;41:166-168.

30. Dwyre DM, Fernando LP, Holland PV. Hepatitis B, hepatitis C and HIV transfusion-transmitted infections in the 21st century. Vox Sang 2011;100:92-98.

31. https://www.who.int/en/news-room/fact-sheets/detail/blood-safety-and-availability. Accessed 26th Apr 2020.

32. Ghosh K, Mishra K. Nucleic acid amplification testing in Indian blood banks: A review with perspectives. Indian J Pathol Microbiol 2017;60:313-318.

33. Tonorezos ES, Barnea D, Cohn RJ, Cypriano MS, Fresneau BC, Haupt R, Hjorth L, Ishida Y, Kruseova J, Kuehni CE, Kurkure PA, Langer T, Nathan PC, Skeen JE, Skinner R, Tacyildiz N, van den Heuvel-Eibrink MM, Winther JF, Hudson MM, Oeffinger KC. Models of Care for Survivors of Childhood Cancer From Across the Globe: Advancing Survivorship Care in the Next Decade. J Clin Oncol 2018;36:2223-2230.

34. Children's Oncology Group Nursing Discipline Clinical Practice Subcommittee/Survivorship in collaboration with the Late Effects Committee. Establishing and Enhancing Services for Childhood Cancer Survivors: Long-Term Follow-Up Program Resource Guide. Children's Oncology Group; 2007; Available on-line: www.survivorshipguidelines.org.

35. Scottish Intercollegiate Guidelines Network (SIGN). Long term follow up of survivors of childhood cancer. Edinburgh: SIGN; 2013. (SIGN publication no. 132). [March 2013]. Available from URL: http://www.sign.ac.uk.

36. Haupt R, Essiaf S, Dellacasa C, Ronckers CM, Caruso S, Sugden E, Zadravec Zaletel L, Muraca M, Morsellino V, Kienesberger A, Blondeel A, Saraceno D, Ortali M, Kremer LCM, Skinner R, Roganovic J, Bagnasco F, Levitt GA, De Rosa M, Schrappe M, Hjorth L, Ladenstein R; PanCareSurFup, ENCCA Working Group; ExPo-r-Net Working Group. The 'Survivorship Passport' for childhood cancer survivors. Eur J Cancer 2018;102:69-81.

37. Poplack DG, Fordis M, Landier W, Bhatia S, Hudson MM, Horowitz ME. Childhood cancer survivor care: development of the Passport for Care. Nat Rev Clin Oncol 2014;11:740-750.

38. Eshelman-Kent D, Kinahan KE, Hobbie W, Landier W, Teal S, Friedman D, Nagarajan R, Freyer DR. Cancer survivorship practices, services, and delivery: a report from the Children's Oncology Group (COG) nursing discipline, adolescent/young adult, and late effects committees. J Cancer Surviv 2011;5:345-357.

39. Guilcher GM, Fitzgerald C, Pritchard S. A questionnaire based review of long-term follow-up programs for survivors of childhood cancer in Canada. Pediatr Blood Cancer 2009;52:113-115.

40. Signorelli C, Wakefield CE, McLoone JK, Fardell JE, Lawrence RA, Osborn M, Truscott J, Tapp H, Cohn RJ; ANZCHOG Survivorship Study Group. Models of childhood cancer survivorship care in Australia and New Zealand: Strengths and challenges. Asia Pac J Clin Oncol 2017;13:407-415.

41. Essig S, Skinner R, von der Weid NX, Kuehni CE, Michel G. Follow-up programs for childhood cancer survivors in Europe: a questionnaire survey. PLoS One 2012;7:e53201. 
42. Rathore V, Taluja A, Arora PR, Bagai P, Seth R, Kapoor G, Arora RS. Delivery of Services to childhood cancer survivors in India: A national survey. India J Med Ped Oncol. 2020 (In Press).

43. Seth R. The After Treatment Completion Card (ATCC): Medical passport for pediatric cancer survivors. Pediatric Hematology Oncology Journal 2018;3:88-89.

44. McCanney J, Winckworth-Prejsnar K, Schatz AA, Nardi EA, Dwyer AJ, Lieu C, Biru Y, Carlson RW. Addressing Survivorship in Cancer Care. J Natl Compr Canc Netw 2018;16:801-806.

45. Arceci R, Ettinger A, Forman E, Haase GM, Hammond GD, Hoffman R, Kupst MJ, Link MP, Lustig CP, Traynor DS. National action plan for childhood cancer: report of the national summit meetings on childhood cancer. CA Cancer J Clin 2002;52:377-379.

46. https://www.childhoodcancerinternational.org/cci-survivors-network/. Accessed 26th Apr 2020.

47. https://www.acco.org/advocacy/. Accessed 26th Apr 2020.

48. Bhatia S, Armenian SH, Armstrong GT, van Dulmen-den Broeder E, Hawkins MM, Kremer LC, Kuehni CE, Olsen JH, Robison LL, Hudson MM. Collaborative Research in Childhood Cancer Survivorship: The Current Landscape. J Clin Oncol 2015;33:3055-3064.

49. Arora RS, Bakhshi S. Indian pediatric oncology group (InPOG)-collaborative research in India comes of age. Pediatric Hematology Oncology Journal 2016;1:13-17.

\section{Legends}

Figure 1 Front page of the bilingual After Treatment Completion Card at AIIMS (A) and the Survivor Passport at Cankids (B)

Figure 2 (A) KCK survivors at parents support group forum, (B) Ritu Bhalla at the World Health Organization South East Asia Regional Office Workshop, (C) Ugam survivors at the Mumbai Marathon

\section{Hosted file}

PBC Table 1.docx available at https://authorea.com/users/322236/articles/451309-childhoodcancer-survivorship-and-late-effects-the-landscape-in-india-in-2020

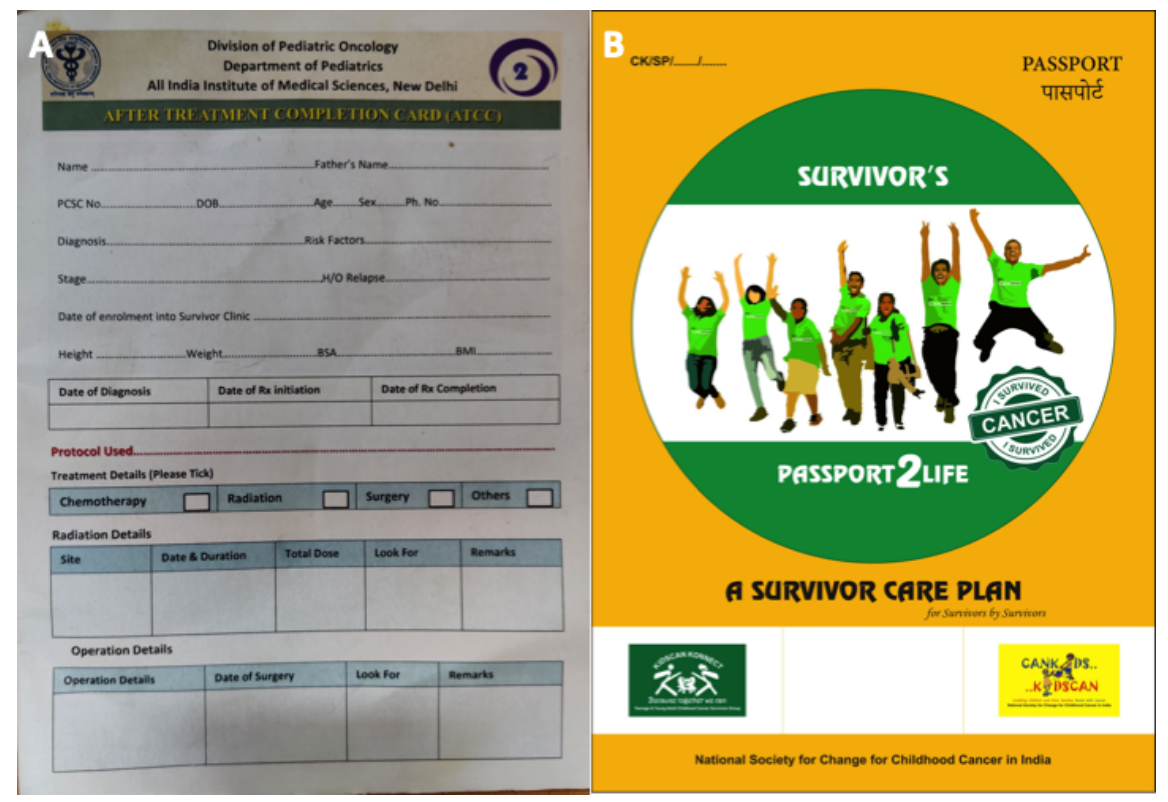




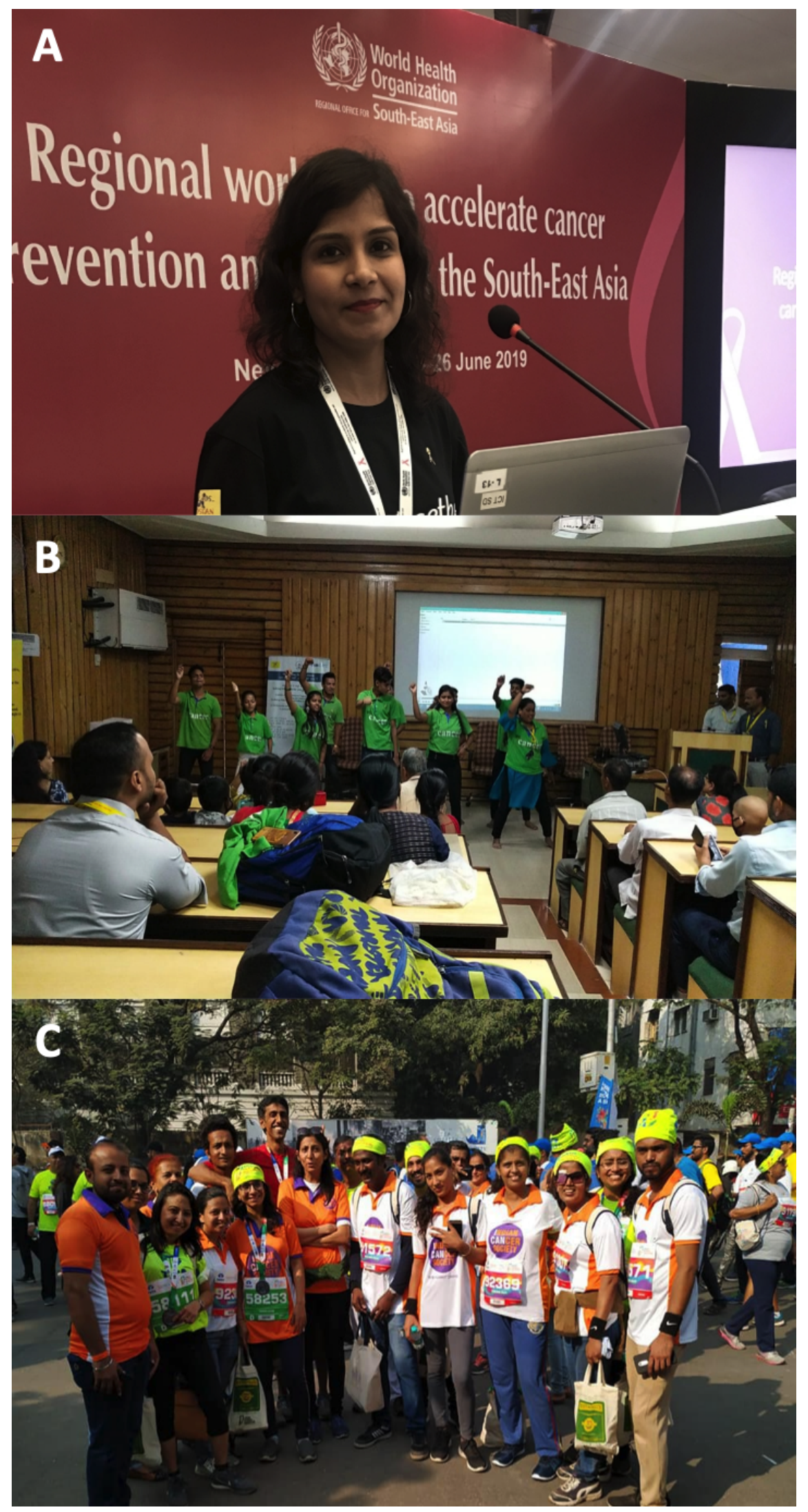

